\title{
Scattering for NLS with a potential on the line
}

\author{
David Lafontaine
}

Laboratoire de Mathématiques J.A. Dieudonné, UMR CNRS 7351, Université de Nice - Sophia Antipolis, 06108 Nice Cedex 02, France

E-mail: david.lafontaine@unice.fr

Abstract. We show the $H^{1}$ scattering for a one dimensional nonlinear Schrödinger equation with a non-negative, repulsive potential $V$ such that $V, x V \in W^{1,1}$, and a mass-supercritical non-linearity. We follow the approach of concentrationcompacity/rigidity first introduced by Kenig and Merle.

Keywords: nonlinear Schrödinger equation, scattering, potential perturbation

\section{Introduction}

We consider the following one dimensional defocusing, non linear Schrödinger equation with a potential

$$
i \partial_{t} u+\Delta u-V u=u|u|^{\alpha}, \quad u(0)=\varphi \in H^{1}(\mathbb{R}) .
$$

If $V \in L^{1},-\Delta+V$ is essentially self-adjoint, so by Stones theorem the equation is globally well posed in $L^{2}(\mathbb{R})$ and $e^{i t(-\Delta+V)}$ is an $L^{2}$-isometry. Goldberg and Schlag obtained in [7] the dispersive estimate

$$
\left\|e^{-i t(-\Delta+V)} \psi\right\|_{L^{\infty}} \lesssim \frac{1}{|t|^{\frac{1}{2}}}\|\psi\|_{L^{1}}
$$

under the assumption that $V$ belongs to $L_{1}^{1}(\mathbb{R})$, i.e. $\int_{-\infty}^{\infty}|V(x)|(1+|x|) d x<\infty$, and that $-\Delta+V$ has no resonance at zero energy. In particular, we will consider a non-negative potential, which always verifies this no-resonance hypothesis as we will see in Section 2. This estimate gives us usual Strichartz estimates described below in the paper. Because of the energy conservation law

$$
E(u(t)):=\frac{1}{2} \int|\nabla u(t)|^{2}+\int V|u(t)|^{2}+\frac{1}{\alpha+2} \int|u(t)|^{\alpha+2}=E(u(0))
$$

the $L^{2}$-well-posedness result extends to the global well-posedness of the problem (1.1) in $H^{1}(\mathbb{R})$ : for every $\varphi \in H^{1}(\mathbb{R})$, there exists a unique, global solution $u \in C\left(\mathbb{R}, H^{1}(\mathbb{R})\right)$ of (1.1). Finally, let us recall that the mass $M(u(t)):=\int|u(t)|^{2}$ is conserved too. 
For the mass-supercritical $(\alpha>4)$ homogeneous equation

$$
i \partial_{t} u+\Delta u=u|u|^{\alpha}, \quad u(0)=\varphi \in H^{1}(\mathbb{R})
$$

it is well known since Nakanishi's paper [12] that the solutions scatter in $H^{1}(\mathbb{R})$, that is, for every solution $u \in C\left(\mathbb{R}, H^{1}(\mathbb{R})\right)$ of (1.2), there exists a unique couple of data $\psi_{ \pm} \in H^{1}(\mathbb{R})$ such that

$$
\left\|u(t)-e^{-i t \Delta} \psi_{ \pm}\right\|_{H^{1}(\mathbb{R})} \underset{t \rightarrow \pm \infty}{\longrightarrow} 0 .
$$

Alternative proofs of this result can be found in [2,5,13] and [14].

We prove the scattering of solutions of (1.1) in dimension one for sufficiently regular, non-negative and repulsive potential $V$.

Theorem 1. Let $\alpha>4$ and $V \in L_{1}^{1}(\mathbb{R})$ be such that $V^{\prime} \in L_{1}^{1}(\mathbb{R})$. We suppose moreover that $V$ is non-negative and repulsive: $V \geqslant 0$ and $x V^{\prime} \leqslant 0$. Then, every solution $u \in C\left(\mathbb{R}, H^{1}(\mathbb{R})\right)$ of (1.1) with potential $V$ scatters in $H^{1}(\mathbb{R})$.

We use the strategy of concentration-compacity/rigidity first introduced by Kenig and Merle in [11], and extented to the intercritical case by Holmer and Roudenko in [8], Duyckaerts, Holmer and Roudenko in [3]. In the case of a potential, the main difficulty is the lack of translation invariance of the equation. Notice that Hong obtained in [9] the same result in the three dimensional case for the focusing equation. However, his approach cannot be extended to lower dimensions, as it requires endpoint Strichartz estimates which are not available. Banica and Visciglia treated in [1] the case of the non linear Schrödinger equation with a Dirac potential on the line, and we follow their approach. The Dirac potential is more singular, but it allows the use of explicit formulas that are not available in the present more general framework.

Remark 1. In dimension one or two, assume that $V$ is smooth and compactly supported, and such that $\int V<0$. Then the operator $-\Delta+V$ has a negative eigenvalue: as a consequence, the hypothesis of positivity of $V$ cannot be relaxed as in dimension three, where [9] only supposes that the potential has a small negative part, and, in the same way, the hypothesis of repulsivity, which is needed for the rigidity, cannot be relaxed to $x V^{\prime}$ having a small positive part.

Remark 2. The hypothesis $V, V^{\prime} \in L^{1}$ are needed to show that the operator $A=-\Delta+V$ verifies the hypothesis of the abstract profile decomposition of [1], whereas the hypothesis $x V^{\prime} \in L^{1}$ and $x V^{\prime} \leqslant 0$ are needed in the rigidity part.

Remark 3. The same proof holds in dimension two up to the numerology and some changes in the Hölder inequalities used in Propositions 6, 7, and 8 to deal with the fact that $H^{1}\left(\mathbb{R}^{2}\right)$ is not embedded in $L^{\infty}\left(\mathbb{R}^{2}\right)$.

Remark 4. In the focusing, mass-supercritical case

$$
i \partial_{t} u+\Delta u-V u+u|u|^{\alpha}=0
$$

the same arguments could be used to prove the scattering up to the natural threshold given by the ground state associated to the equation, in the spirit of [5]. 


\subsection{Notations}

We will denote by $V$ a potential on the line satisfying the hypothesis of Theorem $1, \alpha$ will be a real number such that $\alpha>4$. We set

$$
\begin{aligned}
& H^{1}=H^{1}(\mathbb{R}), \quad C\left(H^{1}\right)=C\left(\mathbb{R}, H^{1}(\mathbb{R})\right), \\
& L^{p} L^{r}=L^{p}\left(\mathbb{R}, L^{r}(\mathbb{R})\right), \quad L^{p}(I) L^{r}=L^{p}\left(I, L^{r}(\mathbb{R})\right)
\end{aligned}
$$

for any interval $I$ of $\mathbb{R}$. We will denote by $\tau_{y}$ the translation operator defined by $\tau_{y} u=u(\cdot-y)$. Finally, we will use $A \lesssim B$ for inequalities of the type $A \leqslant C B$ where $C$ is a universal constant.

\section{Preliminaries}

From now on, we will fix the four following Strichartz exponents

$$
r=\alpha+2, \quad q=\frac{2 \alpha(\alpha+2)}{\alpha^{2}-\alpha-4}, \quad p=\frac{2 \alpha(\alpha+2)}{\alpha+4}, \quad \gamma=\frac{2 \alpha}{\alpha-2} .
$$

\subsection{Strichartz estimates}

Recall that we assume all along the paper that $V$ is in $L_{1}^{1}(\mathbb{R})$ and non negative. Goldberg and Schlag obtained in particular in [7] the dispersive estimate for the Schrödinger operator $-\Delta+V$ under these assumptions.

Indeed, they require the hypothesis of absence of resonances at zero energy. We claim that for $V \geqslant 0$ this hypothesis is satisfied: by the definition of [7], if there is a resonance at zero, the solutions $u_{ \pm}$of

$$
u^{\prime \prime}=V u
$$

such that $u_{ \pm}(x) \rightarrow 1$ as $x \rightarrow \pm \infty$ have a null Wronskian. Therefore $u_{ \pm}$are proportional, so they are both non trivial bounded solutions of (2.1). But such solutions cannot exist: indeed, if $u$ is such a solution, integrating (2.1) one deduces that $u^{\prime}$ has limits at $\pm \infty$. These limits are both zero otherwise $u$ is not bounded. Now, multiplying (2.1) by $u$, integrating it on $[-R, R]$, and letting $R$ going to infinity, we obtain $\int_{\mathbb{R}}\left|u^{\prime}\right|^{2}+V|u|^{2}=0$. Therefore $u=0$, a contradiction.

Proposition 1 (Dispersive estimate [7]). Let $V \in L_{1}^{1}(\mathbb{R})$ be such that $V \geqslant 0$. Then, for all $\psi \in L^{1}(\mathbb{R})$, we have

$$
\left\|e^{-i t(-\Delta+V)} \psi\right\|_{L^{\infty}} \lesssim \frac{1}{|t|^{\frac{1}{2}}}\|\psi\|_{L^{1}}
$$

Note that, interpolating the previous dispersive estimate (2.2) with the mass conservation law, we obtain immediately for all $a \in[2, \infty]$

$$
\left\|e^{i t(-\Delta+V)} \psi\right\|_{L^{a}} \lesssim \frac{1}{|t|^{\frac{1}{2}\left(\frac{1}{a^{\prime}}-\frac{1}{a}\right)}}\|\psi\|_{L^{a^{\prime}}} .
$$


Because of (2.2), we obtain by the classical $T T^{\star}$ method (see for example [10]) the Strichartz estimates

$$
\left\|e^{-i t(-\Delta+V)} \varphi\right\|_{L^{q_{1}} L^{r_{1}}}+\left\|\int_{0}^{t} e^{-i(t-s)(-\Delta+V)} F(s) d s\right\|_{L^{q_{2}} L^{r_{2}}} \lesssim\|\varphi\|_{L^{2}}+\|F\|_{L^{q_{3}^{\prime}} L^{r_{3}^{\prime}}}
$$

for all pairs $\left(q_{i}, r_{i}\right)$ satisfying the admissibility condition in dimension one, that is

$$
\frac{2}{q_{i}}+\frac{1}{r_{i}}=\frac{1}{2}
$$

We will need moreover the following Strichartz estimates associated to non admissible pairs:

Proposition 2 (Strichartz estimates). For all $\varphi \in H^{1}$, all $F \in L^{q^{\prime}} L^{r^{\prime}}$, all $G \in L^{q^{\prime}} L^{r^{\prime}}$ and all $H \in L^{\gamma^{\prime}} L^{1}$

$$
\begin{aligned}
& \left\|e^{-i t(-\Delta+V)} \varphi\right\|_{L^{p} L^{r}} \lesssim\|\varphi\|_{H^{1}} \\
& \left\|e^{-i t(-\Delta+V)} \varphi\right\|_{L^{\alpha} L^{\infty}} \lesssim\|\varphi\|_{H^{1}} \\
& \left\|\int_{0}^{t} e^{-i(t-s)(-\Delta+V)} F(s) d s\right\|_{L^{\alpha} L^{\infty}} \lesssim\|F\|_{L^{q^{\prime} L^{r^{\prime}}}} \\
& \left\|\int_{0}^{t} e^{-i(t-s)(-\Delta+V)} G(s) d s\right\|_{L^{p} L^{r}} \lesssim\|G\|_{L^{q^{\prime}} L^{r^{\prime}}} \\
& \left\|\int_{0}^{t} e^{-i(t-s)(-\Delta+V)} H(s) d s\right\|_{L^{p} L^{r}} \lesssim\|H\|_{L^{\gamma^{\prime}} L^{1}}
\end{aligned}
$$

Proof. The estimates (2.5)-(2.8) are exactly the same as (3.1)-(3.4) of [14], with the operator $-\Delta+V$ instead of $H_{q}$. As the proof of [14] relies only on the admissible Strichartz estimates (2.4) that are given by Proposition 1, the same proof holds here. Finally, (2.9) enters on the frame of the non-admissible inhomogeneous Strichartz estimates of Theorem 1.4 of Foschi's paper [6].

\subsection{Perturbative results}

We will need the three following classical perturbative results, which follow immediately from the previous Strichartz inequalities:

Proposition 3. Let $u \in C\left(H^{1}\right)$ be a solution of (1.1). If $u \in L^{p} L^{r}$, then $u$ scatters in $H^{1}$.

Proposition 4. There exists $\epsilon_{0}>0$, such that, for every data $\varphi \in H^{1}$ such that $\|\varphi\|_{H^{1}} \leqslant \epsilon_{0}$, the corresponding maximal solutions of (1.1) and (1.2) both scatter in $H^{1}$.

Proof of Propositions 3 and 4. The proof is the same as for Propositions 3.1 and 3.2 of [1], using the Strichartz estimates of our Proposition 2 instead of their estimates (3.1), (3.2), (3.3), (3.4). 
Proposition 5. For every $M>0$ there exists $\epsilon>0$ and $C>0$ such that the following occurs. Let $v \in C\left(H^{1}\right) \cap L^{p} L^{r}$ be a solution of the following integral equation with source term $e(t, x)$

$$
v(t)=e^{-i t(\Delta-V)} \varphi-i \int_{0}^{t} e^{-i(t-s)(\Delta-V)}\left(v(s)|v(s)|^{\alpha}\right) d s+e(t)
$$

with $\|v\|_{L^{p} L^{r}}<M$ and $\|e\|_{L^{p} L^{r}}<\epsilon$. Assume moreover that $\varphi_{0} \in H^{1}$ is such that $\left\|e^{-i t(\Delta-V)} \varphi_{0}\right\|_{L^{p} L^{r}}<\epsilon$. Then, the solution $u \in C\left(H^{1}\right)$ to (1.1) with initial condition $\varphi+\varphi_{0}$ satisfies

$$
u \in L^{p} L^{r}, \quad\|u-v\|_{L^{p} L^{r}}<C .
$$

Proof. It is the same as for Proposition 4.7 in [5], using Strichartz estimates (2.8) instead of Strichartztype inequality (4.3) of their paper.

\section{Profile decomposition}

The aim of this section is to show that we can use the abstract profile decomposition obtained by [1], and inspired by [4]:

Theorem (Astract profile decomposition [1]). Let $A: L^{2} \supset D(A) \rightarrow L^{2}$ be a self adjoint operator such that:

- for some positive constants $c, C$ and for all $u \in D(A)$,

$$
c\|u\|_{H^{1}}^{2} \leqslant(A u, u)+\|u\|_{L^{2}}^{2} \leqslant C\|u\|_{H^{1}}^{2},
$$

- let $B: D(A) \times D(A) \ni(u, v) \rightarrow(A u, v)+(u, v)_{L^{2}}-(u, v)_{H^{1}} \in \mathbb{C}$. Then, as $n$ goes to infinity

$$
B\left(\tau_{x_{n}} \psi, \tau_{x_{n}} h_{n}\right) \rightarrow 0 \quad \forall \psi \in H^{1}
$$

as soon as

$$
x_{n} \rightarrow \pm \infty, \quad \sup \left\|h_{n}\right\|_{H^{1}}<\infty
$$

or

$$
x_{n} \rightarrow \bar{x} \in \mathbb{R}, \quad h_{n} \underset{H^{1}}{\rightarrow} 0,
$$

- let $\left(t_{n}\right)_{n \geqslant 1},\left(x_{n}\right)_{n \geqslant 1}$ be sequences of real numbers, and $\bar{t}, \bar{x} \in \mathbb{R}$. Then

$$
\begin{aligned}
& \left|t_{n}\right| \rightarrow \infty \quad \Longrightarrow \quad\left\|e^{i t_{n} A} \tau_{x_{n}} \psi\right\|_{L^{p}} \rightarrow 0, \quad \forall 2<p<\infty, \forall \psi \in H^{1}, \\
& t_{n} \rightarrow \bar{t}, x_{n} \rightarrow \pm \infty \quad \Longrightarrow \quad \forall \psi \in H^{1}, \quad \exists \varphi \in H^{1}, \quad \tau_{-x_{n}} e^{i t_{n} A} \tau_{x_{n}} \psi \stackrel{H^{1}}{\rightarrow} \varphi, \\
& t_{n} \rightarrow \bar{t}, x_{n} \rightarrow \bar{x} \quad \Longrightarrow \quad \forall \psi \in H^{1}, \quad e^{i t_{n} A} \tau_{x_{n}} \psi \stackrel{H^{1}}{\rightarrow} e^{i \bar{t} A} \tau_{\bar{x}} \psi .
\end{aligned}
$$


And let $\left(u_{n}\right)_{n \geqslant 1}$ be a bounded sequence in $H^{1}$. Then, up to a subsequence, the following decomposition holds

$$
u_{n}=\sum_{j=1}^{J} e^{i t_{j}^{n} A} \tau_{x_{n}^{j}} \psi_{j}+R_{n}^{J} \quad \forall J \in \mathbb{N},
$$

where

$$
t_{j}^{n} \in \mathbb{R}, \quad x_{j}^{n} \in \mathbb{R}, \quad \psi_{j} \in H^{1}
$$

are such that

- for any fixed $j$,

$$
\begin{array}{ll}
t_{j}^{n}=0 & \forall n, \text { or } t_{n}^{j} \stackrel{n \rightarrow \infty}{\rightarrow} \pm \infty \\
x_{j}^{n}=0 & \forall n, \text { or } x_{n}^{j} \stackrel{n \rightarrow \infty}{\rightarrow} \pm \infty,
\end{array}
$$

- orthogonality of the parameters:

$$
\left|t_{j}^{n}-t_{k}^{n}\right|+\left|x_{j}^{n}-x_{k}^{n}\right| \stackrel{n \rightarrow \infty}{\rightarrow} \infty, \quad \forall j \neq k,
$$

- decay of the reminder:

$$
\forall \epsilon>0, \exists J \in \mathbb{N}, \quad \limsup _{n \rightarrow \infty}\left\|e^{-i t A} R_{n}^{J}\right\|_{L^{\infty} L^{\infty}} \leqslant \epsilon,
$$

- orthogonality of the Hilbert norm:

$$
\begin{array}{ll}
\left\|u_{n}\right\|_{L^{2}}^{2}=\sum_{j=1}^{J}\left\|\psi_{j}\right\|_{L^{2}}^{2}+\left\|R_{n}^{J}\right\|_{L^{2}}^{2}+o_{n}(1), \quad \forall J \in \mathbb{N} \\
\left\|u_{n}\right\|_{H}^{2}=\sum_{j=1}^{J}\left\|\tau_{x_{n}^{j}} \psi_{j}\right\|_{H}^{2}+\left\|R_{n}^{J}\right\|_{H}^{2}+o_{n}(1), \quad \forall J \in \mathbb{N}
\end{array}
$$

where $(u, v)_{H}=(A u, v)$, and

$$
\left\|u_{n}\right\|_{L^{p}}^{p}=\sum_{j=1}^{J}\left\|e^{i t_{j}^{n} A} \tau_{x_{n}^{j}} \psi_{j}\right\|_{L^{p}}^{p}+\left\|R_{n}^{J}\right\|_{L^{p}}^{p}+o_{n}(1), \quad \forall 2<p<\infty, \forall J \in \mathbb{N} .
$$

We will see that the self-adjoint operator $A:=-\Delta+V$ verifies the hypothesis of the previous theorem.

Proposition 6. Let $A:=-\Delta+V$. Then A satisfies the assumptions (3.1), (3.2), (3.3), (3.4), (3.5). 


\section{Proof.}

Assumption (3.1). Because $V$ is positive and by the Sobolev embedding $H^{1}(\mathbb{R}) \hookrightarrow L^{\infty}$,

$$
\|u\|_{H^{1}}^{2} \leqslant(A u, u)+\|u\|_{L^{2}}=\int|\nabla u|^{2}+\int V|u|^{2}+\int|u|^{2} \leqslant\left(1+\|V\|_{L^{1}}\right)\|u\|_{H^{1}}^{2}
$$

and (3.1) holds.

Assumption (3.2). We have

$$
B\left(\tau_{x_{n}} \psi, \tau_{x_{n}} h_{n}\right)=\int V \tau_{x_{n}} \psi \overline{\tau_{x_{n}} h_{n}} .
$$

If $x_{n} \rightarrow \bar{x} \in \mathbb{R}, h_{n} \underset{H^{1}}{\rightarrow}$, then $\tau_{x_{n}} \psi \rightarrow \tau_{\bar{x}} \psi$ strongly in $L^{2}$ and $V \tau_{x_{n}} h_{n} \rightarrow 0$ weakly in $L^{2}$ (indeed, note that $\left.V \in W^{1,1}(\mathbb{R}) \hookrightarrow L^{2}\right)$, so $B\left(\tau_{x_{n}} \psi, \tau_{x_{n}} h_{n}\right) \rightarrow 0$. Now, let us assume that $x_{n} \rightarrow \pm \infty$ and $\sup \left\|h_{n}\right\|_{H^{1}}<\infty$. For example assume that $x_{n} \rightarrow+\infty . \psi \in H^{1}(\mathbb{R})$ and therefore decays at infinity: $\epsilon>0$ been fixed, we can choose $\Lambda>0$ large enough so that

$$
\sup _{|x| \geqslant \Lambda}|\psi(x)| \leqslant \epsilon
$$

Because $V \in L^{1}, \Lambda$ can also be chosen large enough so that

$$
\int_{|x| \geqslant \Lambda}|V| \leqslant \epsilon
$$

Then, by the Cauchy-Schwarz inequality, and because of the Sobolev embedding $H^{1}(\mathbb{R}) \hookrightarrow L^{\infty}$

$$
\begin{aligned}
\left|B\left(\tau_{x_{n}} \psi, \tau_{x_{n}} h_{n}\right)\right| & \leqslant\left\|h_{n}\right\|_{L^{\infty}} \int\left|V \tau_{x_{n}} \psi\right| \\
& \leqslant \sup _{j \geqslant 1}\left\|h_{j}\right\|_{H^{1}}\left(\int_{\left|x-x_{n}\right| \geqslant \Lambda}\left|V \psi\left(\cdot-x_{n}\right)\right|+\int_{\left|x-x_{n}\right| \leqslant \Lambda}\left|V \psi\left(\cdot-x_{n}\right)\right|\right) .
\end{aligned}
$$

Now, let $n_{0}$ be large enough so that for all $n \geqslant n_{0}, x_{n} \geqslant 2 \Lambda$. Then, for all $n \geqslant n_{0}$

$$
\left|x-x_{n}\right| \leqslant \Lambda \Rightarrow|x| \geqslant \Lambda
$$

and, for all $n \geqslant n_{0}$

$$
\left|B\left(\tau_{x_{n}} \psi, \tau_{x_{n}} h_{n}\right)\right| \leqslant M\left(\epsilon\|V\|_{L^{1}}+\epsilon\|\psi\|_{L^{\infty}}\right)
$$

so (3.2) holds.

Assumption (3.3). It is an immediate consequence of the dispersive estimate and the translation invariance of the $L^{p}$ norms. Indeed, because $H_{0}^{1}(\mathbb{R})=H^{1}(\mathbb{R})$, if $\epsilon>0$, there exists a $C^{\infty}$, compactly supported function $\tilde{\psi}$ such that

$$
\|\tilde{\psi}-\psi\|_{H^{1}} \leqslant \epsilon .
$$


But $\tilde{\psi} \in L^{p^{\prime}}$, so by the dispersive estimate (2.3)

$$
\left\|e^{i t_{n} A} \tau_{x_{n}} \tilde{\psi}\right\|_{L^{p}} \lesssim \frac{1}{\left|t_{n}\right|^{\frac{1}{2}\left(\frac{1}{p^{\prime}}-\frac{1}{p}\right)}}\left\|\tau_{x_{n}} \tilde{\psi}\right\|_{L^{p^{\prime}}}=\frac{1}{\left|t_{n}\right|^{\frac{1}{2}\left(\frac{1}{p^{p}}-\frac{1}{p}\right)}}\|\tilde{\psi}\|_{L^{p^{\prime}}} \rightarrow 0
$$

as $n \rightarrow \infty$. Therefore, for $n$ big enough

$$
\left\|e^{i t_{n} A} \tau_{x_{n}} \tilde{\psi}\right\|_{L^{p}} \leqslant \epsilon .
$$

To achieve the proof, note that $e^{i t A} f$ verifies

$$
\left\|e^{i t A} f\right\|_{H^{1}} \lesssim\|f\|_{H^{1}}
$$

Indeed, as $V$ is positive and in $L^{1}$, by the Sobolev embedding $H^{1}(\mathbb{R}) \hookrightarrow L^{\infty}$ we get

$$
\|\nabla f\|_{L^{2}}^{2} \leqslant\left\|(-\Delta+V)^{\frac{1}{2}} f\right\|_{L^{2}}^{2}=\int|\nabla u|^{2}+\int V|u|^{2} \lesssim\|f\|_{H^{1}} .
$$

So, as $e^{i t A}$ commute with $(-\Delta+V)^{\frac{1}{2}}$ and is an isometry on $L^{2}$,

$$
\begin{aligned}
\left\|e^{i t A} f\right\|_{H^{1}}^{2} & \leqslant\left\|e^{i t A} f\right\|_{L^{2}}^{2}+\left\|(-\Delta+V)^{\frac{1}{2}} e^{i t A} f\right\|_{L^{2}}^{2} \\
& =\left\|e^{i t A} f\right\|_{L^{2}}^{2}+\left\|e^{i t A}(-\Delta+V)^{\frac{1}{2}} f\right\|_{L^{2}}^{2} \\
& =\|f\|_{L^{2}}^{2}+\left\|(-\Delta+V)^{\frac{1}{2}} f\right\|_{L^{2}}^{2} \lesssim\|f\|_{H^{1}}^{2} .
\end{aligned}
$$

Now, because of the Sobolev embedding $H^{1} \hookrightarrow L^{p}$ we obtain using (3.13), (3.14) and (3.15), for $n$ big enough

$$
\begin{aligned}
\left\|e^{i t_{n} A} \tau_{x_{n}} \psi\right\|_{L^{p}} & \leqslant\left\|e^{i t_{n} A} \tau_{x_{n}}(\psi-\tilde{\psi})\right\|_{L^{p}}+\left\|e^{i t_{n} A} \tau_{x_{n}} \tilde{\psi}\right\|_{L^{p}} \\
& \lesssim\left\|e^{i t_{n} A} \tau_{x_{n}}(\psi-\tilde{\psi})\right\|_{H^{1}}+\left\|e^{i t_{n} A} \tau_{x_{n}} \tilde{\psi}\right\|_{L^{p}} \\
& \lesssim\|\psi-\tilde{\psi}\|_{H^{1}}+\left\|e^{i t_{n} A} \tau_{x_{n}} \tilde{\psi}\right\|_{L^{p}} \leqslant 2 \epsilon
\end{aligned}
$$

which achieves the proof of (3.3).

Assumption (3.4). We will show that

$$
t_{n} \rightarrow \bar{t}, x_{n} \rightarrow \pm \infty \quad \Rightarrow \quad\left\|\tau_{-x_{n}} e^{i t_{n}(-\Delta+V)} \tau_{x_{n}} \psi-e^{-i \bar{t} \Delta} \psi\right\|_{H^{1}} \rightarrow 0
$$

and hence (3.4) will hold with $\varphi=e^{-i \bar{t} \Delta} \psi$. As $\tau_{x_{n}}$ is an $H^{1}$ isometry and commute with $e^{-i \bar{t} \Delta}$, it is sufficient to show that, if $t_{n} \rightarrow \bar{t}$ and $x_{n} \rightarrow \pm \infty$, we have

$$
\left\|e^{i t_{n}(-\Delta+V)} \tau_{x_{n}} \psi-e^{-i \bar{t} \Delta} \tau_{x_{n}} \psi\right\|_{H^{1}} \rightarrow 0 .
$$


For example, if $x_{n} \rightarrow+\infty$. Let us first remark that, as $\tau_{x_{n}}$ commutes with $e^{-i \bar{t} \Delta}$ and $e^{-i t_{n} \Delta}$, is an $H^{1}$ isometry, and because $e^{-i t \Delta} \psi \in C\left(H^{1}\right)$

$$
\left\|e^{-i \bar{t} \Delta} \tau_{x_{n}} \psi-e^{-i t_{n} \Delta} \tau_{x_{n}} \psi\right\|_{H^{1}}=\left\|e^{-i \bar{t} \Delta} \psi-e^{-i t_{n} \Delta} \psi\right\|_{H^{1}} \rightarrow 0 .
$$

Hence, decomposing

$$
\begin{aligned}
e^{i t_{n}(-\Delta+V)} \tau_{x_{n}} \psi-e^{-i \bar{t} \Delta} \tau_{x_{n}} \psi= & \left(e^{i t_{n}(-\Delta+V)} \tau_{x_{n}} \psi-e^{-i t_{n} \Delta} \tau_{x_{n}} \psi\right) \\
& +\left(e^{-i t_{n} \Delta} \tau_{x_{n}} \psi-e^{-i \bar{t} \Delta} \tau_{x_{n}} \psi\right)
\end{aligned}
$$

we see that it is sufficient to show that

$$
\left\|e^{i t_{n}(-\Delta+V)} \tau_{x_{n}} \psi-e^{-i t_{n} \Delta} \tau_{x_{n}} \psi\right\|_{H^{1}} \rightarrow 0
$$

Note that $e^{-i t \Delta} \tau_{x_{n}} \psi-e^{i t(-\Delta+V)} \tau_{x_{n}} \psi$ is a solution of the following linear Schrödinger equation with zero initial data

$$
i \partial_{t} u-\Delta u+V u=V e^{-i t \Delta} \tau_{x_{n}} \psi
$$

Therefore, by the inhomogenous Strichartz estimates, as $(4, \infty)$ is admissible in dimension one, and because the translation operator commutes with $e^{-i t \Delta}$, we have for $n$ large enough so that $t_{n} \in(0, \bar{t}+1)$

$$
\begin{aligned}
\left\|e^{i t_{n}(-\Delta+V)} \tau_{x_{n}} \psi-e^{-i t_{n} \Delta} \tau_{x_{n}} \psi\right\|_{L^{2}} & \leqslant\left\|e^{i t(-\Delta+V)} \tau_{x_{n}} \psi-e^{-i t \Delta} \tau_{x_{n}} \psi\right\|_{L^{\infty}(0, \bar{t}+1) L^{2}} \\
& \leqslant\left\|V e^{-i t \Delta} \tau_{x_{n}} \psi\right\|_{L^{\frac{4}{3}}(0, \bar{t}+1) L^{1}} \\
& =\left\|\left(\tau_{-x_{n}} V\right) e^{-i t \Delta} \psi\right\|_{L^{\frac{4}{3}}(0, \bar{t}+1) L^{1}} \\
& \leqslant(\bar{t}+1)^{\frac{3}{4}}\left\|\left(\tau_{-x_{n}} V\right) e^{-i t \Delta} \psi\right\|_{L^{\infty}(0, \bar{t}+1) L^{1}} .
\end{aligned}
$$

Hence, estimating in the same manner the gradient of these quantities, it is sufficient to obtain (3.16) to show that, as $n$ goes to infinity

$$
\left\|\left(\tau_{-x_{n}} V\right) e^{-i t \Delta} \psi\right\|_{L^{\infty}(0, \bar{t}+1) W^{1,1}} \rightarrow 0 .
$$

Let us fix $\epsilon>0 . e^{-i t \Delta} \psi \in C\left([0, \bar{t}+1], H^{1}\right)$ and the functions of $H^{1}(\mathbb{R})$ vanish at infinity, so, using the compacity in time, there exists $\Lambda>0$ such that

$$
\left\|e^{-i t \Delta} \psi\right\|_{L^{\infty}(0, \bar{t}+1) L^{\infty}(|x| \geqslant \Lambda)} \leqslant \epsilon .
$$

On the other hand, as $V \in L^{1}, \Lambda$ can also be taken large enough so that

$$
\int_{|x| \geqslant \Lambda}|V(x)| d x \leqslant \epsilon
$$


Let $n_{0}$ be large enough so that for all $n \geqslant n_{0}, x_{n} \geqslant 2 \Lambda$. Then, for $n \geqslant n_{0}$

$$
\left|x+x_{n}\right| \leqslant \Lambda \Rightarrow|x| \geqslant \Lambda
$$

and for all $t \in(0, \bar{t}+1)$ and all $n \geqslant n_{0}$ we obtain

$$
\begin{aligned}
\left\|\left(\tau_{-x_{n}} V\right) e^{-i t \Delta} \psi\right\|_{L^{1}} & =\int_{\left|x+x_{n}\right| \geqslant \Lambda}\left|V\left(\cdot+x_{n}\right) e^{-i t \Delta} \psi\right|+\int_{\left|x+x_{n}\right| \leqslant \Lambda}\left|V\left(\cdot+x_{n}\right) e^{-i t \Delta} \psi\right| \\
& \leqslant \epsilon\left\|e^{-i t \Delta} \psi\right\|_{L^{\infty}(0, \bar{t}+1) L^{\infty}}+\epsilon\|V\|_{L^{1}} \\
& \leqslant C(\bar{t}, \psi, V) \epsilon
\end{aligned}
$$

thus $\left\|\left(\tau_{-x_{n}} V\right) e^{-i t \Delta} \psi\right\|_{L^{\infty}(0, \bar{t}+1) L^{1}} \rightarrow 0$. With the same argument, because $V^{\prime} \in L^{1}$, we can show that $\left\|\left(\tau_{-x_{n}} V\right)^{\prime} e^{-i t \Delta} \psi\right\|_{L^{\infty}(0, \bar{t}+1) L^{1}} \rightarrow 0$. To obtain (3.17), it only remain to show that

$$
\left\|\tau_{-x_{n}} V\left(e^{-i t \Delta} \psi\right)^{\prime}\right\|_{L^{\infty}(0, \bar{t}+1) L^{1}} \rightarrow 0 .
$$

To this purpose, let $\tilde{\psi}$ be a $C^{\infty}$, compactly supported function such that (recall that we are in dimension one)

$$
\|\psi-\tilde{\psi}\|_{H^{1}} \leqslant \epsilon
$$

We have, by the Cauchy-Schwarz inequality

$$
\begin{aligned}
\left\|\tau_{-x_{n}} V\left(e^{-i t \Delta} \psi\right)^{\prime}\right\|_{L^{1}} & \leqslant\left\|\tau_{-x_{n}} V\left(e^{-i t \Delta} \tilde{\psi}\right)^{\prime}\right\|_{L^{1}}+\left\|\tau_{-x_{n}} V\left(e^{-i t \Delta}(\psi-\tilde{\psi})\right)^{\prime}\right\|_{L^{1}} \\
& \leqslant\left\|\tau_{-x_{n}} V\left(e^{-i t \Delta} \tilde{\psi}\right)^{\prime}\right\|_{L^{1}}+\|V\|_{L^{2}}\left\|\left(e^{-i t \Delta}(\psi-\tilde{\psi})\right)^{\prime}\right\|_{L^{2}} \\
& \leqslant\left\|\tau_{-x_{n}} V\left(e^{-i t \Delta} \tilde{\psi}\right)^{\prime}\right\|_{L^{1}}+\epsilon\|V\|_{L^{2}},
\end{aligned}
$$

where $V \in L^{2}$ because of the Sobolev embedding $W^{1,1}(\mathbb{R}) \hookrightarrow L^{2}(\mathbb{R})$. Then, as $\left(e^{-i t \Delta} \tilde{\psi}\right)^{\prime} \in H^{1}$, $\left\|\tau_{-x_{n}} V\left(e^{-i t \Delta} \tilde{\psi}\right)^{\prime}\right\|_{L^{\infty}(0, \bar{t}+1) L^{1}}$ can be estimated as $\left\|\left(\tau_{-x_{n}} V\right) e^{-i t \Delta} \psi\right\|_{L^{\infty}(0, \bar{t}+1) L^{1}}$, so (3.17) holds and the proof of (3.4) is completed.

Assumption (3.5). We decompose

$$
e^{i t_{n} A} \tau_{x_{n}} \psi-e^{i \bar{t} A} \tau_{\bar{x}} \psi=\left(e^{i t_{n} A} \tau_{x_{n}} \psi-e^{i t_{n} A} \tau_{\bar{x}} \psi\right)+\left(e^{i t_{n} A} \tau_{\bar{x}} \psi-e^{i \bar{t} A} \tau_{\bar{x}} \psi\right) .
$$

On the one hand, using the estimate (3.15)

$$
\left\|e^{i t_{n} A} \tau_{x_{n}} \psi-e^{i t_{n} A} \tau_{\bar{x}} \psi\right\|_{H^{1}} \lesssim\left\|\tau_{x_{n}} \psi-\tau_{\bar{x}} \psi\right\|_{H^{1}} \underset{n \rightarrow \infty}{\longrightarrow} 0
$$

by the Lebesgue's dominated convergence theorem. On the other hand,

$$
\left\|e^{i t_{n} A} \tau_{\bar{x}} \psi-e^{i \bar{t} A} \tau_{\bar{x}} \psi\right\|_{H^{1}} \underset{n \rightarrow \infty}{\longrightarrow} 0
$$

because $e^{i \cdot A} \tau_{\bar{x}} \psi \in C\left(H^{1}\right)$, and the last assumption is verified. 


\section{Non linear profiles}

In this section, we will see that for a data which escapes to infinity, the solutions of (1.1) and (1.2) are the same, in the sense given by the three following Propositions.

Propositions 7, 8 and 9 are the analogous of Propositions 3.4 and 3.6 of [1]. The non linear Schrödinger equation with a Dirac potential is more singular, but it allows the use of explicit formulas that are not available in the present more general framework.

Proposition 7. Let $\psi \in H^{1},\left(x_{n}\right)_{n} \geqslant 1 \in \mathbb{R}^{\mathbb{N}}$ be such that $\left|x_{n}\right| \rightarrow \infty$. Then, up to a subsequence

$$
\left\|e^{-i t \Delta} \tau_{x_{n}} \psi-e^{-i t(\Delta-V)} \tau_{x_{n}} \psi\right\|_{L^{p} L^{r}} \rightarrow 0
$$

as $n \rightarrow \infty$.

Proof. Up to a subsequence, we can assume that $x_{n} \rightarrow+\infty$ or $x_{n} \rightarrow-\infty$. Let us assume for example $x_{n} \rightarrow+\infty$.

As a first step, we will show that

$$
\sup _{n \in \mathbb{N}}\left\|e^{i t(-\Delta+V)} \tau_{x_{n}} \psi\right\|_{L^{p}(T, \infty) L^{r}} \rightarrow 0
$$

as $T \rightarrow \infty$. Pick $\epsilon>0$. There exists a $C^{\infty}$, compactly supported function $\tilde{\psi}$ such that

$$
\|\tilde{\psi}-\psi\|_{H^{1}} \leqslant \epsilon
$$

By Strichartz estimates

$$
\left\|e^{i t(-\Delta+V)}\left(\tau_{x_{n}} \tilde{\psi}-\tau_{x_{n}} \psi\right)\right\|_{L^{p} L^{r}} \lesssim\left\|\tau_{x_{n}} \tilde{\psi}-\tau_{x_{n}} \psi\right\|_{H^{1}}=\|\tilde{\psi}-\psi\|_{H^{1}} \leqslant \epsilon .
$$

On the other hand, as $\tau_{x_{n}} \tilde{\psi} \in L^{r^{\prime}}$ the dispersive estimate (2.3) gives us

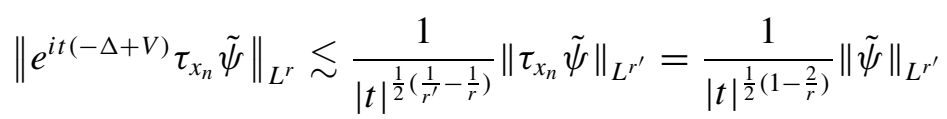

but $\frac{p}{2}\left(1-\frac{2}{r}\right)=\frac{\alpha^{2}}{\alpha+4}>1$ and $t \rightarrow \frac{1}{|t|^{\frac{1}{2}\left(1-\frac{2}{r}\right)}} \in L^{q}(1, \infty)$. So, there exists $T>0$ such that

$$
\sup _{n \in \mathbb{N}}\left\|e^{i t(-\Delta+V)} \tau_{x_{n}} \tilde{\psi}\right\|_{L^{p}(|t| \geqslant T) L^{r}} \leqslant \epsilon
$$

Taking $\tau_{x_{n}} \psi=\tau_{x_{n}} \tilde{\psi}+\left(\tau_{x_{n}} \psi-\tau_{x_{n}} \tilde{\psi}\right)$, we then obtain for $T>0$ large enough

$$
\sup _{n \in \mathbb{N}}\left\|e^{i t(-\Delta+V)} \tau_{x_{n}} \psi\right\|_{L^{p}(|t| \geqslant T) L^{r}} \lesssim \epsilon
$$

and (4.2) holds. 
To obtain (4.1), we are now reduced to show that for $T>0$ fixed

$$
\left\|e^{-i t \Delta} \tau_{x_{n}} \psi-e^{i t(-\Delta+V)} \tau_{x_{n}} \psi\right\|_{L^{p}(0, T) L^{r}} \rightarrow 0
$$

as $n \rightarrow \infty$. Let $\epsilon>0 . e^{-i t \Delta} \tau_{x_{n}} \psi-e^{i t(-\Delta+V)} \tau_{x_{n}} \psi$ is a solution of the following linear Schrödinger equation with zero initial data

$$
i \partial_{t} u-\Delta u+V u=V e^{-i t \Delta} \tau_{x_{n}} \psi .
$$

So, by the inhomogenous Strichartz estimate (2.9)

$$
\begin{aligned}
& \left\|e^{-i t \Delta} \tau_{x_{n}} \psi-e^{i t(-\Delta+V)} \tau_{x_{n}} \psi\right\|_{L_{t}^{p}(0, T) L^{r}} \lesssim\left\|V e^{-i t \Delta} \tau_{x_{n}} \psi\right\|_{L_{t}^{\gamma^{\prime}}(0, T) L^{1}} \\
& \lesssim T^{\frac{1}{\gamma^{\prime}}}\left\|V e^{-i t \Delta} \tau_{x_{n}} \psi\right\|_{L^{\infty}(0, T) L^{1}} \\
& =T^{\frac{1}{\gamma^{\prime}}}\left\|\left(\tau_{-x_{n}} V\right) e^{-i t \Delta} \psi\right\|_{L^{\infty}(0, T) L^{1}}
\end{aligned}
$$

because the translation operator $\tau_{x_{n}}$ commutes with the propagator $e^{-i t \Delta}$. But

$$
\left\|\left(\tau_{-x_{n}} V\right) e^{-i t \Delta} \psi\right\|_{L^{\infty}(0, T) L^{1}} \underset{n \rightarrow \infty}{\longrightarrow} 0
$$

as seen in the proof of Proposition 6, point (3.4).

Proposition 8. Let $\psi \in H^{1},\left(x_{n}\right)_{n \geqslant 1} \in \mathbb{R}^{\mathbb{N}}$ be such that $\left|x_{n}\right| \rightarrow \infty, U \in C\left(H^{1}\right) \cap L^{p} L^{r}$ be the unique solution to (1.2) with initial data $\psi$, and $U_{n}(t, x):=U\left(t, x-x_{n}\right)$. Then, up to a subsequence

$$
\left\|\int_{0}^{t} e^{-i(t-s) \Delta}\left(U_{n}\left|U_{n}\right|^{\alpha}\right)(s) d s-\int_{0}^{t} e^{-i(t-s)(\Delta-V)}\left(U_{n}\left|U_{n}\right|^{\alpha}\right)(s) d s\right\|_{L^{p} L^{r}} \rightarrow 0
$$

as $n \rightarrow \infty$.

Proof. We follow the same spirit of proof as for Proposition 7. We begin to show that

$$
\sup _{n \in \mathbb{N}}\left\|\int_{0}^{t} e^{-i(t-s)(\Delta-V)}\left(U_{n}\left|U_{n}\right|^{\alpha}\right)(s) d s\right\|_{L^{p}([T, \infty)) L^{r}} \rightarrow 0
$$

as $T$ goes to infinity.

We decompose

$$
\begin{aligned}
\left\|\int_{0}^{t} e^{-i(t-s)(\Delta-V)}\left(U_{n}\left|U_{n}\right|^{\alpha}\right)(s) d s\right\|_{L^{p}([T, \infty)) L^{r}} & \leqslant \int_{0}^{T} e^{-i(t-s)(\Delta-V)}\left(U_{n}\left|U_{n}\right|^{\alpha}\right)(s) d s \|_{L^{p}([T, \infty)) L^{r}} \\
& +\left\|\int_{T}^{t} e^{-i(t-s)(\Delta-V)}\left(U_{n}\left|U_{n}\right|^{\alpha}\right)(s) d s\right\|_{L^{p}([T, \infty)) L^{r}}
\end{aligned}
$$


where, by the inhomogenous Strichartz estimates

$$
\left\|\int_{T}^{t} e^{-i(t-s)(\Delta-V)}\left(U_{n}\left|U_{n}\right|^{\alpha}\right)(s) d s\right\|_{L^{p}\left([T, \infty) L^{r}\right)} \leqslant\left\|U_{n}\left|U_{n}\right|^{\alpha}\right\|_{L^{q^{\prime}\left([T, \infty) L^{\left.r^{\prime}\right)}\right.}}=\left\|U|U|^{\alpha}\right\|_{L^{q^{\prime}\left([T, \infty) L^{r^{\prime}}\right)}}
$$

and, by the Hölder inequality

$$
\left\|U|U|^{\alpha}\right\|_{L^{q^{\prime}}\left([T, \infty) L^{r^{\prime}}\right)} \leqslant\|U\|_{L^{p}\left([T, \infty) L^{r}\right)}^{\alpha+1} \underset{T \rightarrow \infty}{\longrightarrow} 0
$$

independently of $n$. On the other hand, by the dispersive estimate (2.3)

$$
\begin{aligned}
& \left\|\int_{0}^{T} e^{-i(t-s)(\Delta-V)}\left(U_{n}\left|U_{n}\right|^{\alpha}\right)(s) d s\right\|_{L^{p}[T, \infty) L^{r}} \\
& \quad \leqslant\left\|\int_{0}^{T}\right\| e^{-i(t-s)(\Delta-V)}\left(U_{n}\left|U_{n}\right|^{\alpha}\right)(s)\left\|_{L^{r}} d s\right\|_{L^{p}([T, \infty))} \\
& \quad \lesssim\left\|\int_{0}^{T}(t-s)^{-\frac{1}{2}\left(1-\frac{2}{r}\right)}\right\|\left(U_{n}\left|U_{n}\right|^{\alpha}\right)(s)\left\|_{L^{r^{\prime}}} d s\right\|_{L^{p}([T, \infty))} \\
& \quad=\left\|\int_{0}^{T}(t-s)^{-\frac{1}{2}\left(1-\frac{2}{r}\right)}\right\|\left(U|U|^{\alpha}\right)(s)\left\|_{L^{r^{\prime}}} d s\right\|_{L^{p}([T, \infty))} \longrightarrow 0 \\
& \quad \leqslant\left\|\int_{\mathbb{R}}|t-s|^{-\frac{1}{2}\left(1-\frac{2}{r}\right)}\right\|\left(U|U|^{\alpha}\right)(s)\left\|_{L^{r^{\prime}}} d s\right\|_{L^{p}([T, \infty))} \longrightarrow 0
\end{aligned}
$$

as $T$ goes to infinity. Indeed, note that by the Hardy-Littlewood-Sobolev inequality

$$
\left\|\int_{\mathbb{R}}|t-s|^{-\frac{1}{2}\left(1-\frac{2}{r}\right)}\right\|\left(U|U|^{\alpha}\right)(s)\left\|_{L^{r^{\prime}}} d s\right\|_{L^{p}} \lesssim\left\|U|U|^{\alpha}\right\|_{L^{q^{\prime}} L^{r^{\prime}}} \leqslant\|U\|_{L^{p} L^{r}}^{\alpha+1}<\infty
$$

so (4.4) holds. The same estimate is obviously valid for the propagator $e^{-i t \Delta}$.

It remains to show that for $T>0$ fixed,

$$
\left\|\int_{0}^{t} e^{-i(t-s) \Delta}\left(U_{n}\left|U_{n}\right|^{\alpha}\right) d s-\int_{0}^{t} e^{-i(t-s)(\Delta-V)}\left(U_{n}\left|U_{n}\right|^{\alpha}\right) d s\right\|_{L^{p}(0, T) L^{r}} \rightarrow 0
$$

as $n \rightarrow \infty$. The difference

$$
\int_{0}^{t} e^{-i(t-s) \Delta}\left(U_{n}\left|U_{n}\right|^{\alpha}\right) d s-\int_{0}^{t} e^{-i(t-s)(\Delta-V)}\left(U_{n}\left|U_{n}\right|^{\alpha}\right) d s
$$

is the solution of the following linear Schrödinger equation, with zero initial data

$$
i \partial_{t} u-\Delta u+V u=V \int_{0}^{t} e^{-i(t-s) \Delta}\left(U_{n}\left|U_{n}\right|^{\alpha}\right) d s .
$$


As a consequence, by the Strichartz estimate (2.9)

$$
\begin{aligned}
& \left\|\int_{0}^{t} e^{-i(t-s) \Delta}\left(U_{n}\left|U_{n}\right|^{\alpha}\right) d s-\int_{0}^{t} e^{-i(t-s)(\Delta-V)}\left(U_{n}\left|U_{n}\right|^{\alpha}\right) d s\right\|_{L^{p}(0, T) L^{r}} \\
& \quad \lesssim\left\|V \int_{0}^{t} e^{-i(t-s) \Delta}\left(U_{n}\left|U_{n}\right|^{\alpha}\right) d s\right\|_{L^{\gamma^{\prime}(0, T) L^{1}}} \\
& \quad \lesssim T \frac{1}{\gamma^{\prime}}\left\|\left(\tau_{-x_{n}} V\right) \int_{0}^{t} e^{-i(t-s) \Delta}\left(U|U|^{\alpha}\right) d s\right\|_{L^{\infty}(0, T) L^{1}}
\end{aligned}
$$

But $\int_{0}^{t} e^{-i(t-s) \Delta}\left(U|U|^{\alpha}\right) d s \in C\left([0, T], H^{1}\right)$ and the functions of $H^{1}(\mathbb{R})$ vanish at infinity, so there exists $\Lambda>0$ such that

$$
\left\|\int_{0}^{t} e^{-i(t-s) \Delta}\left(U|U|^{\alpha}\right) d s\right\|_{L^{\infty}(0, T) L^{\infty}(|x| \geqslant \Lambda)} \leqslant \epsilon
$$

so

$$
\left\|\left(\tau_{-x_{n}} V\right) \int_{0}^{t} e^{-i(t-s) \Delta}\left(U|U|^{\alpha}\right) d s\right\|_{L^{\infty}(0, T) L^{1}} \underset{n \rightarrow \infty}{\longrightarrow} 0
$$

in the same way as in the proof of Proposition 6, point (3.4).

Proposition 9. Let $\psi \in H^{1},\left(x_{n}\right)_{n} \geqslant 1,\left(t_{n}\right)_{n} \geqslant 1 \in \mathbb{R}^{\mathbb{N}}$ be such that $\left|x_{n}\right| \rightarrow \infty$ and $t_{n} \rightarrow \pm \infty, U$ be a solution to (1.2) such that

$$
\left\|U(t)-e^{-i t \Delta} \psi\right\|_{H^{1}} \underset{t \rightarrow \pm \infty}{\longrightarrow} 0
$$

and $U_{n}(t, x):=U\left(t-t_{n}, x-x_{n}\right)$. Then, up to a subsequence

$$
\left\|e^{-i\left(t-t_{n}\right) \Delta} \tau_{x_{n}} \psi-e^{-i\left(t-t_{n}\right)(\Delta-V)} \tau_{x_{n}} \psi\right\|_{L^{p} L^{r}} \rightarrow 0
$$

and

$$
\left\|\int_{0}^{t} e^{-i(t-s) \Delta}\left(U_{n}\left|U_{n}\right|^{\alpha}\right) d s-\int_{0}^{t} e^{-i(t-s)(\Delta-V)}\left(U_{n}\left|U_{n}\right|^{\alpha}\right) d s\right\|_{L^{p} L^{r}} \rightarrow 0
$$

as $n \rightarrow \infty$.

Proof. The proof is the same as for Proposition 7 and Proposition 8, decomposing the time interval in $\left\{\left|t-t_{n}\right|>T\right\}$ and its complementary.

Finally, we will need the following Proposition of non linear scattering: 
Proposition 10. Let $\varphi \in H^{1}$. Then there exists $W_{ \pm} \in C\left(H^{1}\right) \cap L_{\mathbb{R}^{ \pm}}^{p} L^{r}$, solution of $(1.1)$ such that

$$
\left\|W_{ \pm}(t, \cdot)-e^{-i t(\Delta-V)} \varphi\right\|_{H^{1}} \underset{t \rightarrow \pm \infty}{\longrightarrow} 0
$$

moreover, if $t_{n} \rightarrow \mp \infty$ and

$$
\varphi_{n}=e^{-i t_{n}(\Delta-V)} \varphi, \quad W_{ \pm, n}(t)=W_{ \pm}\left(t-t_{n}\right)
$$

then

$$
W_{ \pm, n}(t)=e^{-i t(\Delta-V)} \varphi_{n}+\int_{0}^{t} e^{-i(t-s)(\Delta-V)}\left(W_{ \pm, n}\left|W_{ \pm, n}\right|^{\alpha}\right)(s) d s+f_{ \pm, n}(t)
$$

where

$$
\left\|f_{ \pm, n}\right\|_{L_{\mathbb{R}^{ \pm}}^{p} L^{r}} \underset{n \rightarrow \infty}{\longrightarrow} 0 .
$$

Proof. The same proof as [1], Proposition 3.5, holds, as it involves only the analogous Strichartz estimates.

\section{Construction of a critical element}

We have now all the tools to extract a critical element following the approach of [5]. Let

$$
E_{c}=\sup \left\{E>0 \mid \forall \varphi \in H^{1}, E(\varphi)<E \Rightarrow \text { the solution of (1.1) with data } \varphi \text { is in } L^{p} L^{r}\right\} .
$$

We will suppose that the critical energy $E_{c}$ is finite, and deduce the existence of a solution of (1.1) with a relatively compact flow in $H^{1}$.

Proposition 11. If $E_{c}<\infty$, then there exists $\varphi_{c} \in H^{1}, \varphi_{c} \neq 0$, such that the corresponding solution $u_{c}$ of (1.1) verifies that $\left\{u_{c}(t), t \geqslant 0\right\}$ is relatively compact in $H^{1}$.

Proof. Because of Proposition 4, $E_{c}>0$. Therefore, if $E_{c}<\infty$, there exists a sequence $\varphi_{n}$ of non-zero elements of $H^{1}$, such that, if we denote by $u_{n} \in C\left(H^{1}\right)$ the corresponding solution of (1.1), we have

$$
E\left(\varphi_{n}\right) \underset{n \rightarrow \infty}{\longrightarrow} E_{c}
$$

and

$$
u_{n} \notin L^{q} L^{r} .
$$

Thanks to the Proposition 6, we can apply the abstract profile decomposition of [1] to the $H^{1}$-bounded sequence $\varphi_{n}$ and the operator $A=-\Delta+V$. Up to a subsequence, $\varphi_{n}$ writes, for all $J \in \mathbb{N}$ :

$$
\varphi_{n}=\sum_{j=1}^{J} e^{-i t_{j}^{n}(-\Delta+V)} \tau_{x_{j}^{n}} \psi_{j}+R_{n}^{J},
$$


where $t_{j}^{n}, x_{j}^{n}, \psi_{j}, R_{n}^{J}$ verifies (3.6)-(3.12). From (3.11) and (3.12), we have

$$
E_{c} \geqslant \limsup _{n \rightarrow \infty} \sum_{j=1}^{J} E\left(e^{-i t_{j}^{n}(-\Delta+V)} \tau_{x_{j}^{n}} \psi_{j}\right) .
$$

We show that there is exactly one non trivial profile, that is $J=1$. By contradiction, assume that $J>1$. To each profile $\psi_{j}$ we associate family of non linear profiles $\left(U_{j, n}\right)_{n \geqslant 0}$. Let $j \in\{1, \ldots, J\}$. We are in exactly one of the following situations:

(1) If $\left(t_{j}^{n}, x_{j}^{n}\right)=(0,0)$. By the orthogonality condition, notice that this can happen only for one profile. Because $J>1$, we have $E\left(\psi_{j}\right)<E_{c}$, so the solution of (1.1) with data $\psi_{j}$ scatters. If this case happens, let $N \in C\left(H^{1}\right) \cap L^{p} L^{r}$ be this solution, otherwise, we set $N=0$.

(2) If $t_{j}^{n}=0$ and $\left|x_{j}^{n}\right| \rightarrow \infty$. Let $U_{j} \in C\left(H^{1}\right) \cap L^{p} L^{r}$ be the unique solution to (1.2) with initial data $\psi_{j}$. We set $U_{n, j}(x, t):=U\left(x-x_{j}^{n}, t\right)$.

(3) If $x_{j}^{n}=0$ and $t_{j}^{n} \rightarrow \pm \infty$. By Proposition 10 , there exists $U_{j} \in C_{\mathbb{R}_{ \pm}}\left(H^{1}\right) \cap L_{\mathbb{R}_{ \pm}}^{p} L^{r}$ a solution to (1.1) such that

$$
\left\|U_{j}(t)-e^{-i t(\Delta-V)} \psi_{j}\right\|_{H^{1}} \underset{t \rightarrow \pm \infty}{\longrightarrow} 0
$$

and verifying (4.7), (4.8), (4.9), (4.10). We have

$$
E\left(U_{j}\right)=\lim _{n \rightarrow \infty} E\left(e^{-i t_{j}^{n}(-\Delta+V)} \tau_{x_{j}^{n}} \psi_{j}\right)<E_{c}
$$

so $U_{j} \in L^{q} L^{r}$. We set $U_{j, n}(t, x):=U_{j}\left(t-t_{j}^{n}, x\right)$.

(4) If $\left|x_{j}^{n}\right| \rightarrow \infty$ and $t_{j}^{n} \rightarrow \pm \infty$. Let $U_{j} \in C\left(H^{1}\right) \cap L^{p} L^{r}$ be a solution to (1.2) such that

$$
\left\|U_{j}(t)-e^{-i t \Delta} \psi_{j}\right\|_{H^{1}} \underset{t \rightarrow \pm \infty}{\longrightarrow} 0 .
$$

We set $U_{j, n}(t, x):=U_{j}\left(t-t_{j}^{n}, x-x_{j}^{n}\right)$.

Now, let

$$
Z_{n, J}:=N+\sum_{j} U_{n, j}
$$

By the results of the non linear profiles section - Propositions 7 and 8 in situation (2), Proposition 9 in situation (3) and Proposition 10 in situation (4), we have

$$
\begin{aligned}
Z_{n, J}= & e^{-i t(\Delta-V)}\left(\varphi_{n}-R_{n, J}\right)+\int_{0}^{t} e^{-i(t-s)(\Delta-V)}\left(N|N|^{\alpha}\right)(s) d s \\
& +\sum_{j} \int_{0}^{t} e^{-i(t-s)(\Delta-V)}\left(U_{j, n}\left|U_{j, n}\right|^{\alpha}\right)(s) d s+r_{n, J}
\end{aligned}
$$


with

$$
\left\|r_{n, J}\right\|_{L^{p} L^{r}} \rightarrow 0
$$

as $n \rightarrow \infty$. The decomposition (5.1) is the same as obtained in the proof of Proposition 4.1 of [1], and we therefore obtain the critical element following their proof, using our perturbative result of Proposition 5 instead of their Proposition 3.3, and the Strichartz inequalities of our Proposition 2 instead of estimates (3.1), (3.2), (3.3), (3.4) of their paper.

\section{Rigidity}

In this section, we will show that the critical solution constructed in the previous one assuming the fact that $E_{c}<\infty$ cannot exist.

We will need the following classical result concerning the compact families of $H^{1}$

Proposition 12. Suppose that $\{u(t), t \geqslant 0\}$ is relatively compact in $H^{1}$. Then, for any $\epsilon>0$, there exists $R>0$ such that

$$
\sup _{t \geqslant 0} \int_{|x| \geqslant R}\left(|\nabla u(t, x)|^{2}+|u(t, x)|^{2}+|u(t, x)|^{\alpha+2}\right) d x \leqslant \epsilon .
$$

Proof. Classic, see e.g. [5].

Now, we can show the rigidity Proposition needed to end the proof:

Proposition 13. Suppose that $u \in C\left(H^{1}\right)$ is a solution of (1.1) such that $\{u(t), t \geqslant 0\}$ is relatively compact in $H^{1}$. Then $u=0$.

Proof. By a classical elementary computation, we get the following virial identities:

Lemma 1. Let $u \in C\left(H^{1}\right)$ be a solution to (1.1) and $\chi$ be a compactly supported, regular function. Then

$$
\begin{aligned}
& \partial_{t} \int \chi|u|^{2}=2 \operatorname{Im} \int \chi^{\prime} u^{\prime} \bar{u} \\
& \partial_{t}^{2} \int \chi|u|^{2}=4 \int \chi^{\prime \prime}\left|u^{\prime}\right|^{2}+\frac{2 \alpha}{\alpha+2} \int \chi^{\prime \prime}|u|^{\alpha+2}-2 \int \chi^{\prime} V^{\prime}|u|^{2}-\int \chi^{(4)}|u|^{2} .
\end{aligned}
$$

Now, we assume by contradiction that $u \neq 0$. Let $\chi \in C_{c}^{\infty}$ be such that $\chi(x)=x^{2}$ for $|x| \leqslant 1$ and $\chi(x)=0$ for $|x| \geqslant 2$, set $\chi_{R}:=R^{2} \chi(\dot{\bar{R}})$ and

$$
z_{R}(t)=\int \chi_{R}|u(t)|^{2}
$$


we have, by (6.1), the Cauchy-Schwarz inequality and the conservation of energy

$$
\left|z_{R}^{\prime}(t)\right| \leqslant 2 \int\left|\chi_{R}^{\prime}\right|\left|u^{\prime}\right||\bar{u}| \leqslant C E(u)^{\frac{1}{2}} M(u)^{\frac{1}{2}} R .
$$

Moreover, by (6.2)

$$
\begin{aligned}
z_{R}^{\prime \prime}(t)= & 4 \int \chi_{R}^{\prime \prime}\left|u^{\prime}\right|^{2}+\frac{2 \alpha}{\alpha+2} \int \chi_{R}^{\prime \prime}|u|^{\alpha+2}-2 \int \chi_{R}^{\prime} V^{\prime}|u|^{2}-\int \chi_{R}^{(4)}|u|^{2} \\
\geqslant & 8 \int_{|x| \leqslant R}\left|u^{\prime}\right|^{2}+\frac{4 \alpha}{\alpha+2} \int_{|x| \leqslant R}|u|^{\alpha+2}-C \int_{|x|>R}\left(|u|^{2}+|u|^{\alpha+2}+\left|u^{\prime}\right|^{2}\right) \\
& -2 \int \chi_{R}^{\prime} V^{\prime}|u|^{2}-\int \chi^{(4)}|u|^{2}
\end{aligned}
$$

but, because of conservation of the mass

$$
\left.\left|\int \chi^{(4)}\right| u\right|^{2} \mid \leqslant \frac{C}{R^{2}}\|u(0)\|_{L^{2}}
$$

and, because $V$ is repulsive (i.e. $x V^{\prime} \leqslant 0$ ), using the Cauchy-Schwarz inequality, the Sobolev injection $H^{1} \hookrightarrow L^{\infty}$ and the conservation laws

$$
\begin{aligned}
-2 \int \chi_{R}^{\prime} V^{\prime}|u|^{2} & =-2 \int_{|x| \leqslant R} x V^{\prime}|u|^{2}+2 \int_{|x|>R} \chi_{R}^{\prime} V^{\prime}|u|^{2} \\
& \geqslant-C \int_{|x|>R}\left|x V^{\prime}\right||u|^{2} \geqslant-C\left\|x V^{\prime}\right\|_{L^{1}(|x|>R)}\|u\|_{L^{\infty}}^{2} \\
& \geqslant-C\left\|x V^{\prime}\right\|_{L^{1}(|x|>R)}\|u\|_{H^{1}}^{2} \geqslant-C(u(0))\left\|x V^{\prime}\right\|_{L^{1}(|x|>R)} .
\end{aligned}
$$

Let $R_{0}$ be large enough so that

$$
\int_{|x| \leqslant R_{0}}|u|^{\alpha+2} \geqslant \frac{1}{2} \int|u|^{\alpha+2}:=\delta .
$$

We have $\delta>0$ because we suppose that $u$ is non zero. For $R \geqslant R_{0}$, we obtain combining (6.4) with (6.5), (6.6), and (6.7)

$$
z_{R}^{\prime \prime}(t) \geqslant C\left(\delta-\int_{|x|>R}\left(|u|^{2}+|u|^{\alpha+2}+\left|u^{\prime}\right|^{2}\right)-\frac{1}{R^{2}}\|u(0)\|_{L^{2}}-\left\|x V^{\prime}\right\|_{L^{1}(|x|>R)}\right) .
$$

Because $x V^{\prime} \in L^{1}$ and using the compacity hypothesis combined with Proposition 12, there exists $R \geqslant R_{0}$ large enough so that

$$
\int_{|x|>R}\left(|u|^{2}+|u|^{\alpha+2}+\left|u^{\prime}\right|^{2}\right)+\frac{1}{R^{2}}\|u(0)\|_{L^{2}}+\left\|x V^{\prime}\right\|_{L^{1}(|x|>R)} \leqslant \frac{\delta}{2}
$$


then, (6.8) gives

$$
z_{R}^{\prime \prime}(t) \geqslant \frac{C \delta}{2}>0
$$

Integrating this last inequality contradicts (6.3) as $t \rightarrow \infty$.

We are now in position to end the proof of Theorem 1:

Proof of Theorem 1. If $E_{c}<\infty$, then the Proposition 11 allows us to extract a critical element $\varphi_{c} \in$ $H^{1}, \varphi_{c} \neq 0$, such that the corresponding solution $u_{c}$ of (1.1) verifies that $\left\{u_{c}(t), t \geqslant 0\right\}$ is relatively compact in $H^{1}$. By Proposition 13, such a critical solution cannot exist, so $E_{c}=\infty$ and by Proposition 3, all the solutions of (1.1) scatter in $H^{1}$.

\section{Acknowledgements}

The author thanks N. Visciglia for having submitted him this problem, enlighting discussions and his warm welcome in Pisa, J. Zheng for his helpfull comments, his Ph.D. advisor F. Planchon for his disponibility and advices, and the referee for his careful reading and his constructive criticism.

\section{References}

[1] V. Banica and N. Visciglia, Scattering for NLS with a delta potential, J. Differential Equations 260(5) (2016), 4410-4439. doi:10.1016/j.jde.2015.11.016.

[2] J. Colliander, M. Grillakis and N. Tzirakis, Tensor products and correlation estimates with applications to nonlinear Schrödinger equations, Comm. Pure Appl. Math. 62(7) (2009), 920-968. doi:10.1002/cpa.20278.

[3] T. Duyckaerts, J. Holmer and S. Roudenko, Scattering for the non-radial 3D cubic nonlinear Schrödinger equation, Math. Res. Lett. 15(6) (2008), 1233-1250. doi:10.4310/MRL.2008.v15.n6.a13.

[4] L. Fanelli and N. Visciglia, The lack of compactness in the Sobolev-Strichartz inequalities, J. Math. Pures Appl. (9) 99(3) (2013), 309-320. doi:10.1016/j.matpur.2012.06.015.

[5] D. Fang, J. Xie and T. Cazenave, Scattering for the focusing energy-subcritical nonlinear Schrödinger equation, Sci. China Math. 54(10) (2011), 2037-2062. doi:10.1007/s11425-011-4283-9.

[6] D. Foschi, Inhomogeneous Strichartz estimates, J. Hyperbolic Differ. Equ. 2(1) (2005), 1-24. doi:10.1142/ S0219891605000361.

[7] M. Goldberg and W. Schlag, Dispersive estimates for Schrödinger operators in dimensions one and three, Comm. Math. Phys. 251(1) (2004), 157-178. doi:10.1007/s00220-004-1140-5.

[8] J. Holmer and S. Roudenko, A sharp condition for scattering of the radial 3D cubic nonlinear Schrödinger equation, Comm. Math. Phys. 282(2) (2008), 435-467. doi:10.1007/s00220-008-0529-y.

[9] Y. Hong, Scattering for a nonlinear Schrödinger equation with a potential, Commun. Pure Appl. Anal. 15 (2016), 15711601, doi:10.3934/cpaa.2016003.

[10] M. Keel and T. Tao, Endpoint Strichartz estimates, Amer. J. Math. 120(5) (1998), 955-980, available at: http://muse.jhu. edu/journals/american_journal_of_mathematics/v120/120.5keel.pdf. doi:10.1353/ajm.1998.0039.

[11] C.E. Kenig and F. Merle, Global well-posedness, scattering and blow-up for the energy-critical, focusing, non-linear Schrödinger equation in the radial case, Invent. Math. 166(3) (2006), 645-675. doi:10.1007/s00222-006-0011-4.

[12] K. Nakanishi, Energy scattering for nonlinear Klein-Gordon and Schrödinger equations in spatial dimensions 1 and 2 , J. Funct. Anal. 169(1) (1999), 201-225. doi:10.1006/jfan.1999.3503.

[13] F. Planchon and L. Vega, Bilinear virial identities and applications, Ann. Sci. Éc. Norm. Supér. (4) 42(2) (2009), $261-290$.

[14] N. Visciglia, On the decay of solutions to a class of defocusing NLS, Math. Res. Lett. 16(5) (2009), 919-926. doi:10.4310/ MRL.2009.v16.n5.a14. 